Article

\title{
Potential of the Endophytic Fungus Phialocephala fortinii Rac56 Found in Rhodiola Plants to Produce Salidroside and $p$-Tyrosol
}

\author{
Jinlong Cui, Tingting Guo, Jianbin Chao, Mengliang Wang * and Junhong Wang \\ Institute of Applied Chemistry, Shanxi University, Taiyuan 030006, China; CJL717@sxu.edu.cn (J.C.); \\ guott880118@163.com (T.G.); chao@sxu.edu.cn (J.C.); wangjunh@sxu.edu.cn (J.W.) \\ * Correspondence: mlwang@sxu.edu.cn; Tel.: +86-351-7016101 \\ Academic Editor: Marcello Iriti \\ Received: 25 February 2016; Accepted: 6 April 2016; Published: 16 April 2016
}

\begin{abstract}
Hydroxyphenyl)ehyl- $\beta$-D-glucopyranoside (salidroside) and 4-(2-hydroxyethyl)phenol ( $p$-tyrosol) are famous food and medicine additives originally derived from alpine Rhodiola plants. Salidroside or $p$-tyrosol production by the endophytic fungus Rac56 (Phialocephala fortinii) was confirmed by UPLC/Q-TOF-MS and ${ }^{1} \mathrm{H}-\mathrm{NMR}$. The fermentation conditions were optimized by orthogonal design using data processing system software. The broth fermentation results showed that salidroside and $p$-tyrosol extraction yields from Rac56 were stable and reached $1.729 \pm 0.06 \mathrm{mg}$ and $1.990 \pm 0.05 \mathrm{mg}$ per $\mathrm{mL}$ of culture medium, respectively. The optimal conditions for salidroside and $p$-tyrosol production in fermentation culture of Rac56 were determined to be $25^{\circ} \mathrm{C}$, $\mathrm{pH}$ values of 7 and 5, Czapek-Dox culture medium volumes of $150 \mathrm{~mL}$ and $50 \mathrm{~mL}$ in $250 \mathrm{~mL}$ flasks, rotation speeds of $100 \times \mathrm{g}$ and $200 \times \mathrm{g}$, and fermentation durations of 7 and 15 days, respectively. Under these optimal conditions, stable yields of $2.339 \pm 0.1093 \mathrm{mg}$ and $2.002 \pm 0.0009 \mathrm{mg}$ per $\mathrm{mL}$ of culture medium of salidroside and $p$-tyrosol, respectively, were obtained, indicating that the P. fortinii Rac56 strain is a promising source of these compounds.
\end{abstract}

Keywords: Phialocephala fortinii; fungal bioactivity; Rhodiola angusta; accumulation of active component

\section{Introduction}

Salidroside (SAL) and $p$-tyrosol (TYR) (Figure 1) are the main bioactive ingredients of all Rhodiola spp., such as $R$. rosea and $R$. crenulata [1], which is a rare and valuable plant of the Crassulaceae family found in high-altitude ( $>2500 \mathrm{~m}$ ) regions [2]. It was used as food crop and folk medicine by many ancient civilizations because of its pharmacologic effects, such as antioxidant, anti-fatigue, anti-aging, as well as adaptogenic properties [3]. With increasing demand for SAL and TYR as additives in the health food, cosmetic, and pharmaceutical industries, wild Rhodiola plants are being over-exploited [4]. However, artificial cultivation of Rhodiola plants has been limited by the elevation requirement of planting areas, long production cycle, and low content of active components [4]. In recent years, tissue and cell culture as well as chemical synthesis methods have been used for SAL and TYR production; however, these methods are costly, low yielding, and conducive to pollution [5]. As a result, the prices of SAL and TYR are high and their supplies are limited.

Endophytes are microorganisms that live specifically within the tissue of their host plants without causing apparent disease symptoms [6]. Studies have shown that all investigated plants harbor endophytic fungi $[7,8]$. During long-term co-evolution, complex interaction and cross-talk between endophytic fungi and their hosts, such as signal exchange and horizontal gene transmission [9,10], have occurred to jointly cope with biotic and abiotic stresses [11,12]. Since a taxol-producing endophytic fungus Taxomyces andreanae was isolated from its host plant Taxus brevifolia in 1993 [13], follow-up 
studies have further confirmed that endophytic fungi and their host plant might have the same synthetic routes of some secondary metabolites and could produce similar or identical metabolites [14]. In recent years, many plant-derived bioactive products have been isolated from endophytic fungi [15], making them more economical and easier to produce at a larger scale by submerged fermentation.

In the course of our continuous search for endophytic fungi in the three main species of Rhodiola in China, a total of 347 isolates were obtained for the first time, from which SAL and TYR-producing fungi were screened. Four isolates were found to be potential candidates, but only one isolate Rac56 was determined to be a remarkable producer of SAL and TYR. The current study is the first to report Rhodiola endophytic fungus and optimize its fermentation conditions, with results showing that the isolate could serve as a reliable source for SAL and TYR production.

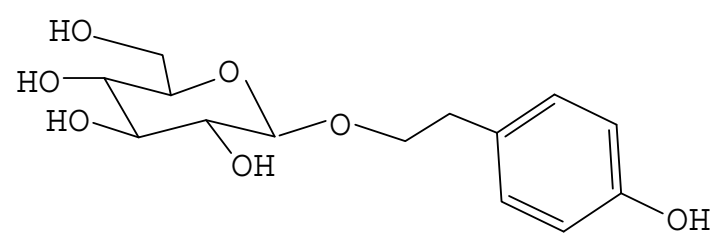

(a)

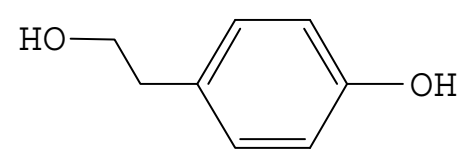

(b)

Figure 1. Chemical structures of two active compounds in Rhodiola plants. (a) Salidroside; (b) p-tyrosol.

\section{Results}

\subsection{Isolation and Screening of SAL- and TYR-Producing Fungi}

A total of 347 endophytic fungi were obtained from three Rhodiola species, and about 180 representative morphotypes $(71,57$, and 52 isolates from $R$. crenulata, $R$. angusta, and $R$. sachalinensis, respectively) were fermented to determine the SAL and TYR content. The high performance liquid chromography (HPLC) analyses results showed that four endophytic fungi (Rac12, Rac56, and Rac63 from $R$. angusta; Rct30 from $R$. crenulata) can produce SAL or TYR (Table 1). Figure S1 shows the ultraviolet absorption and HPLC chromatograms of SAL and TYR standards, and the extracts of Rac12 (mycelial extract), Rct30 (mycelial extract), Rac56 and Rac63 (filtrate extract), respectively. Rac56 could produce an average of $1.729 \pm 0.063 \mathrm{mg}$ SAL and $1.990 \pm 0.050 \mathrm{mg}$ TYR, and each of the other three isolates produced an average of $\leqslant 0.402 \pm 0.248 \mathrm{mg}$ SAL or TYR per mL of culture medium (Table 1 ). Furthermore, production of SAL and TYR from most isolates declined even after being sub-cultured several times in the follow-up studies; only Rac56 remained the most stable and had the highest yield, with the yield in culture filtrate shown to be higher than that in mycelia. Therefore, the fermentation broth extract of Rac56 was chosen for further studies.

Table 1. SAL and TYR production from endophytic fungi (Rhodiola plants).

\begin{tabular}{|c|c|c|c|c|c|c|c|c|}
\hline Name & \multicolumn{2}{|c|}{$\operatorname{Rac12}(\mathrm{mg} / \mathrm{mL})$} & \multicolumn{2}{|c|}{ Rct30 (mg/mL) } & \multicolumn{2}{|c|}{ Rac56 (mg/mL) } & \multicolumn{2}{|c|}{ Rac63 (mg/mL) } \\
\hline SAL $^{1}$ & - & $0.131 \pm 0.009$ & - & $0.343 \pm 0.465$ & $1.729 \pm 0.063$ & $0.028 \pm 0.032$ & $0.402 \pm 0.248$ & - \\
\hline
\end{tabular}

\subsection{Identification of Rac56 as the Ideal SAL- and TYR-Producing Fungus}

The colony diameter of Rac56 was $45 \mathrm{~mm}$ after inoculation in the PDA medium at $25 \pm 2{ }^{\circ} \mathrm{C}$ for 20 days, with gray-black color, central uplift, neat edge, no visible secretion in medium, and a black melanized outer edge. The mycelia were dense, appeared floss-shaped, and had no evident substrate mycelium (Figure 2a). Under a microscope, the hypha was dark colored and had transverse septa, which belong to dark septate endophytes (DSE), had a diameter range of 1.5-4.5 $\mu \mathrm{m}$, abundant 
globular particle contents in hypha with terminal expansion, and did not produce conidium (Figure 2b). The identification was authenticated by ITS analyses. The rDNA sequence of Rac56 (KJ542292, 519 bp) and its closest related species available at GenBank were selected for construction of NJ phylogenetic tree using MEGA 4.0 program after sequence alignment with Clustal W. The endophytic fungus Rac56 was found to be closely related to Phialocephala fortinii (AY394915 and AY394921) (Figure 2c) with $99 \%$ homology. The morphological characteristic described above corresponded to anamorphic P. fortinii [16-20], which led to its designation as P. fortinii.

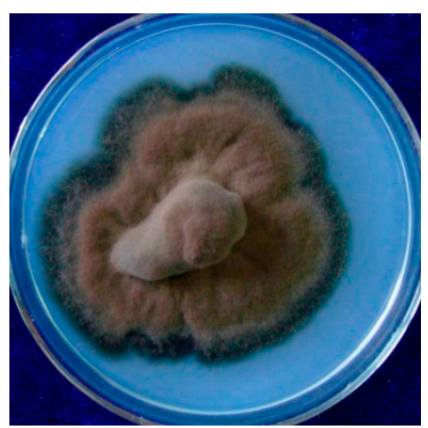

(a)

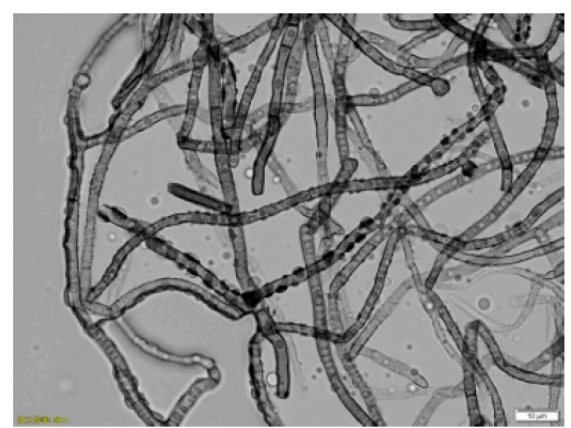

(b)

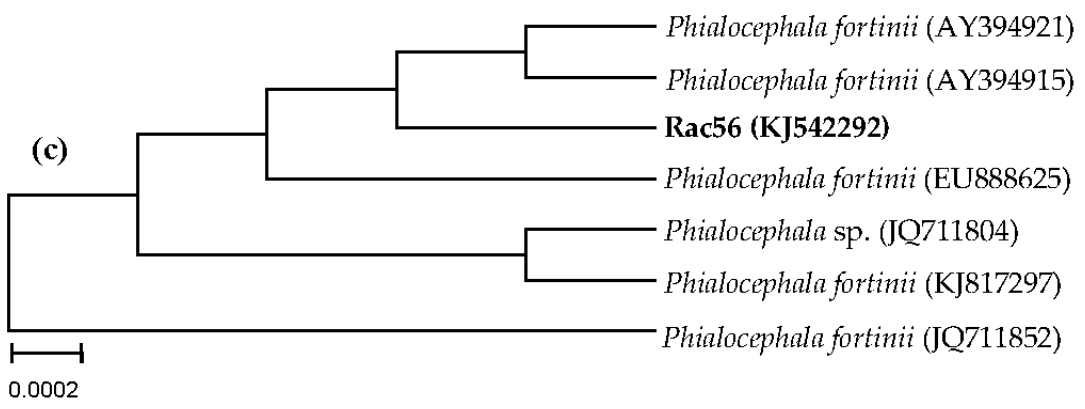

Figure 2. Identification of endophytic fungus Rac56 from Rhodiola angusta. Morphological analyses of the colony characteristics (a) and fungal mycelium (b) were conducted 20 days post culturing. Under a microscope, the mycelia were observed to be dark with a size range of 1.5-4.5 $\mu \mathrm{m}$. Aneighbor-joining tree (c) based on ITS rDNA sequence of Rct56 and its closest ITS rDNA matches in GenBank was constructed using MEGA 4.0 software based on 1000 bootstrap replicates.

\subsection{Validation of SAL and TYR Produced from Rac56 by LC-MS and ${ }^{1} H-N M R$}

SAL and TYR produced by the Rac56 strain were further identified by UPLC/Q-TOF-MS. Results showed that the retention times (Rt) of the two metabolites produced by Rac56 were $2.15 \mathrm{~min}$ and $2.51 \mathrm{~min}$ (Figure 3a), corresponding to $2.14 \mathrm{~min}$ and $2.49 \mathrm{~min}$ of standard SAL and TYR in the UV profile (Figure 3b), respectively; the molecular ion at $m / z 299.1130[\mathrm{M}-\mathrm{H}]^{-}$and $\mathrm{m} / z 345.1194$ $[\mathrm{M}-\mathrm{H}+\mathrm{HCOOH}]^{-}$of the chemical component produced by Rac56 (Figure 3c) was consistent with the molecular ion at $m / z 299.1128[\mathrm{M}-\mathrm{H}]^{-}$and $m / z 345.1185[\mathrm{M}-\mathrm{H}+\mathrm{HCOOH}]^{-}$of standard SAL (Figure 3d).

However, molecular ion peaks of TYR could not be obtained from the MS spectrum by using either negative-ion or positive ion mode although it could be detected by the UV profile of both the TYR standard and the Rac56 sample. The fungal SAL and TYR produced by the Rac56 strain were examined by ${ }^{1} \mathrm{H}-\mathrm{NMR}$ spectroscopic analysis. The aromatic region $\left(\delta_{\mathrm{H}} 6.5-7.2 \mathrm{ppm}, \mathrm{H} 3 / 5\right.$ and $\mathrm{H} 2 / 6$ proton signals for SAL and TYR were $6.982 \mathrm{ppm}$ to $7.009 \mathrm{ppm})$ and the sugar region $\left(\delta_{\mathrm{H}} 3-4.5 \mathrm{ppm}\right.$, $\mathrm{H}^{-1}$ proton signal for SAL was $4.218 \mathrm{ppm}$ to $4.248 \mathrm{ppm}$ ) were found to contain aromatic compound and glycosidic constituent peaks identical to those found in commercially available SAL and TYR, respectively (Figure 4).These findings are also consistent with previous studies [4,21] and lend further support to the authenticity of SAL and TYR produced by the Rac56 strain. 

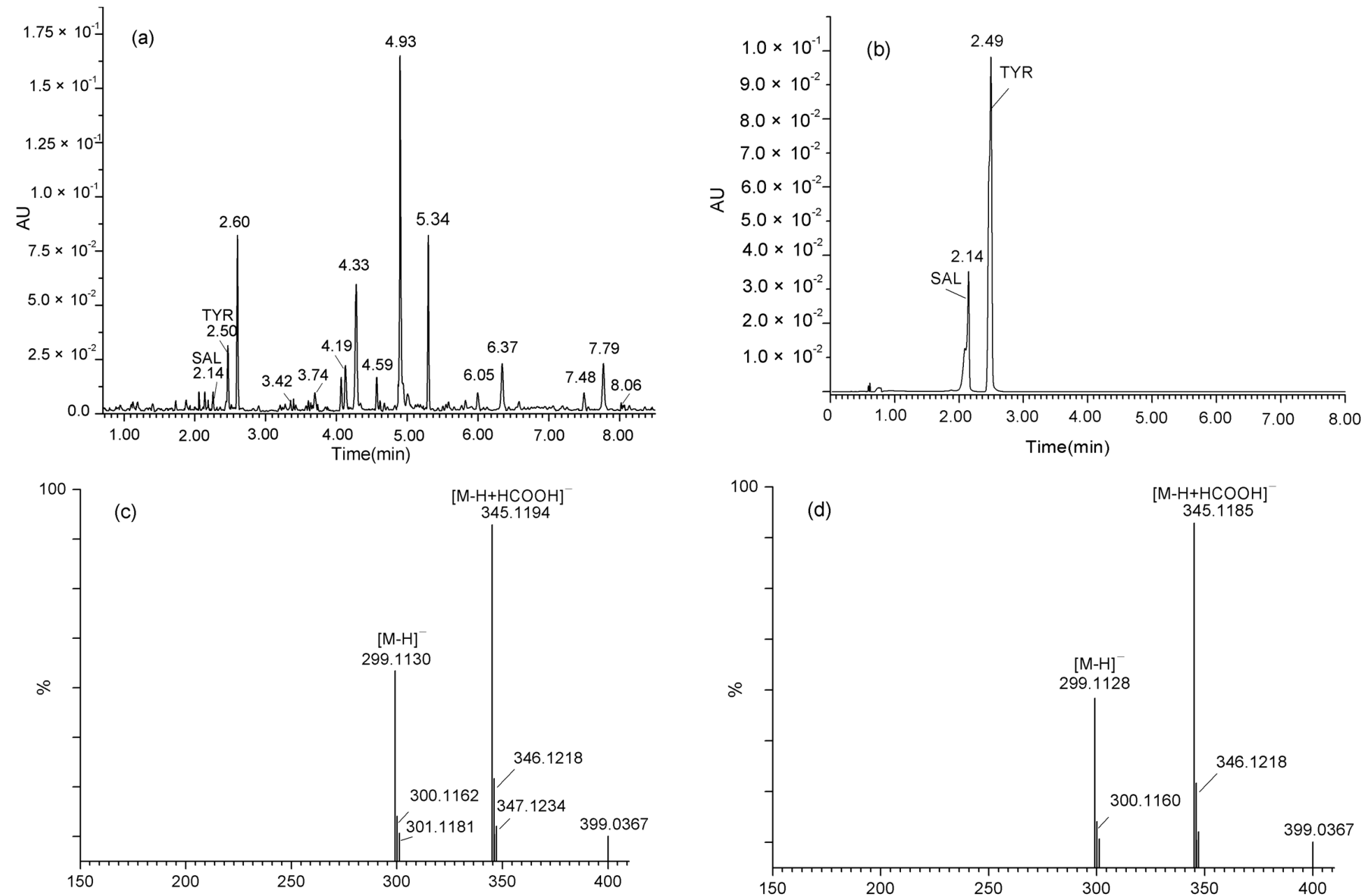

Figure 3. UV profile and MS chromatogram of the culture filtrate the Rac56 strain and commercially available salidroside (SAL) and p-tyrosol (TYR). (a) UV chromatograms of culture filtrate of fungus Rac56 containing SAL (Rt, 2.15) and TYR (Rt, 2.51) detected at a wavelength of $277 \mathrm{~nm}$; (b) UV chromatograms of authentic SAL (Rt, 2.14) and TYR (Rt, 2.49) monitored by UPLC-UV detector at the wavelength of $277 \mathrm{~nm}$; (c) Molecular ion peak at $m / z 299.1130[\mathrm{M}-\mathrm{H}]^{-}$and $m / z 345.1194$ $[\mathrm{M}-\mathrm{H}+\mathrm{HCOOH}]^{-}$of SAL produced by Rac56; (d) Molecular ion peak $[\mathrm{M}-\mathrm{H}]^{-}$at $m / z 299.1128$ and $[\mathrm{M}-\mathrm{H}+\mathrm{HCOOH}]^{-}$at $m / z 345.1185$ of authentic SAL. 


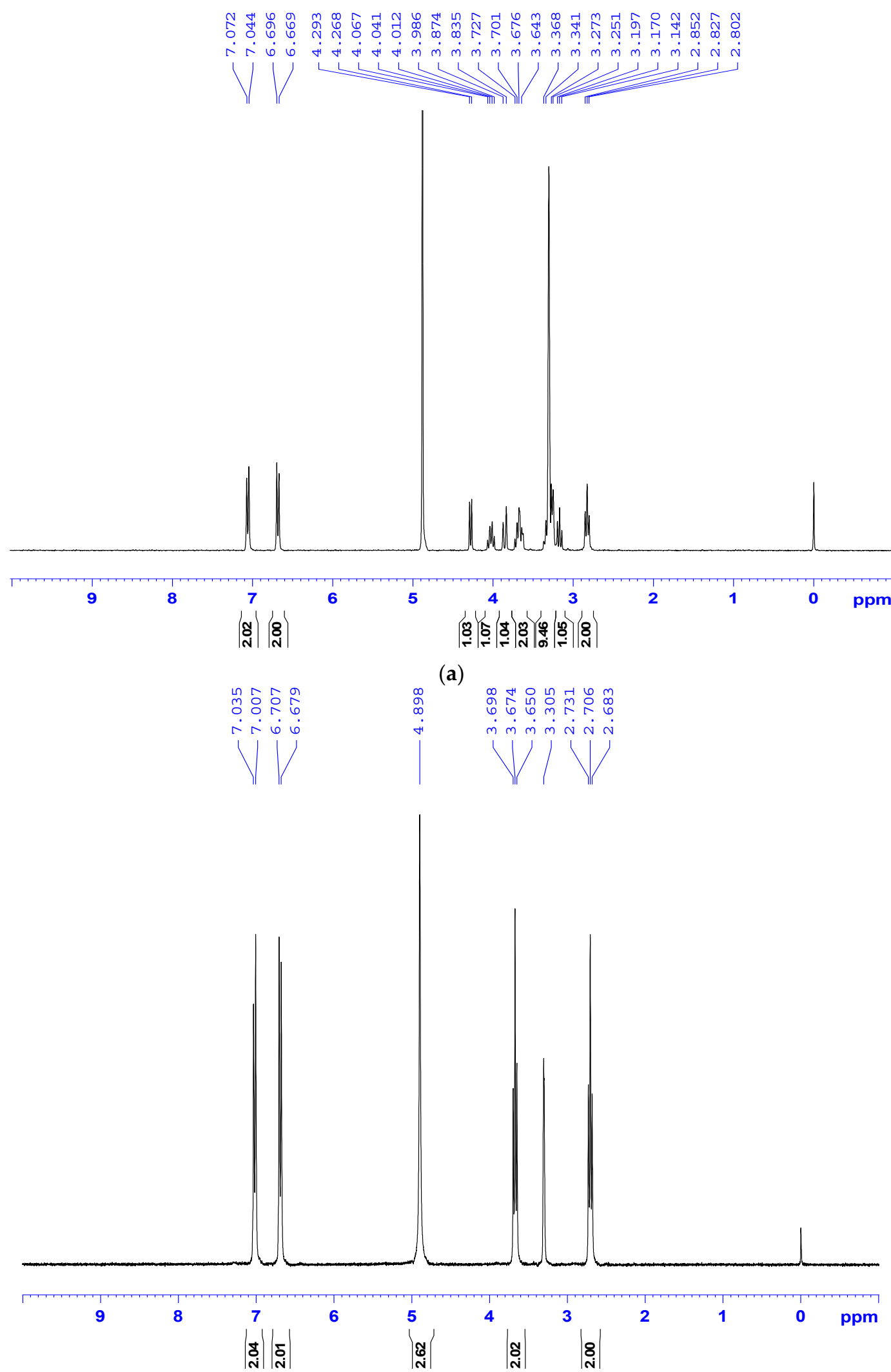

(b)

Figure 4. Cont. 


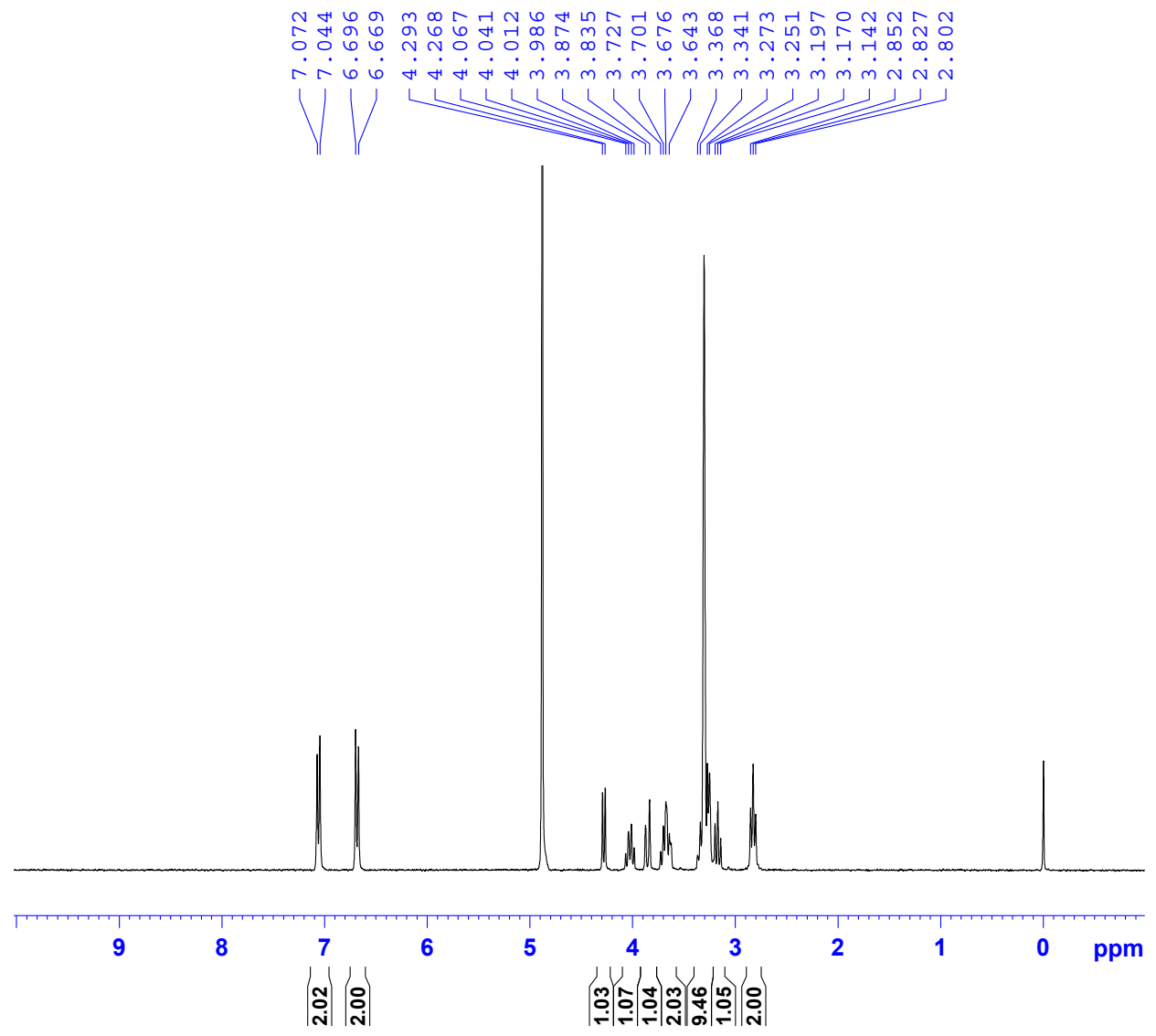

(c)

Figure 4. ${ }^{1} \mathrm{H}-\mathrm{NMR}$ spectra of samples. ${ }^{1} \mathrm{H}-\mathrm{NMR}$ spectra of standard/commercially available SAL (a) and TYR (b); and the chemicals in culture filtrate extract from Rac56 (c). All test samples were normalized to solvent $\mathrm{CD}_{3} \mathrm{OD}$ signal, in accordance with [4,21].

\subsection{Fermentation and Optimal Growth Conditioning for SAL and TYR Production from Rac56}

Results of HPLC quantification of SAL and TYR indicated that the yields of SAL cultured with Czapek-Dox medium (CD), potato dextrose medium (PD), Sabouraud medium (SD), and lactose-beef extract-peptone medium (LBP) media were $1.715 \pm 0.007 \mathrm{mg} / \mathrm{mL}, 1.368 \pm 0.021 \mathrm{mg} / \mathrm{mL}$, $1.046 \pm 0.018 \mathrm{mg} / \mathrm{mL}$ and $1.140 \pm 0.023 \mathrm{mg} / \mathrm{mL}$, respectively, and the yields of TYR in CD $(1.863 \pm 0.041 \mathrm{mg} / \mathrm{mL})$ was higher than that in PD $(1.551 \pm 0.030 \mathrm{mg} / \mathrm{mL}), \mathrm{SD}(1.419 \pm 0.008 \mathrm{mg} / \mathrm{mL})$, or LBP $(1.067 \pm 0.016 \mathrm{mg} / \mathrm{mL})$. Thus, the CD medium was chosen as the best medium for subsequent optimization of the fermentation conditions.

Tables 2-4 present the SAL and TYR production optimization using a five factor and four levels $\left(\mathrm{L} 16\left(4^{5}\right)\right)$ orthogonal experimental design. The results of $F$ test showed that temperature, culture volume, and fermentation time had significant effects on SAL and TYR production. The significance of each factor was determined by the corresponding $F$ values and $P$ values (Tables 2 and 3). $p$ values of $p \leqslant 0.05$ and $p \leqslant 0.01$ were considered statistically significant and highly significant, respectively (Tables 2 and 3). Based on the highest values of SAL production, the optimal fermentation conditions were $25^{\circ} \mathrm{C}$, initial $\mathrm{pH} 7.0,150 \mathrm{~mL}$ for culture volume in a $250 \mathrm{~mL}$ flask, $100 \times \mathrm{g}$ of rotation speed, and seven days of fermentation time. The optimal condition for TYR production was set as; temperature of $25^{\circ} \mathrm{C}$, initial $\mathrm{pH}$ of 5.0 , culture volume of $50 \mathrm{~mL}$ in a $250 \mathrm{~mL}$ flask, rotation speed of $200 \times \mathrm{g}$, and fermentation time of 15 days. With the optimal conditions outlined above, SAL and TYR yields of $2.3392 \pm 0.1093 \mathrm{mg}$ and $2.0018 \pm 0.0009 \mathrm{mg}$ per $\mathrm{mL}$ of culture medium, respectively, were obtained. These yields were higher than those of any other conditions (Table 4). 
Table 2. Test of significant effects of five factors on fermentation for SAL production from Rac56 using Data Processing System.

\begin{tabular}{cccccccccccc}
\hline \multicolumn{10}{c}{${ }^{1}$ SAL } \\
\hline $\mathrm{T}_{1}$ & 19.298 & 19.086 & 19.471 & 19.1383 & 18.4791 & ${ }^{2} \mathrm{~V}$ & $\mathrm{SS}$ & $\mathrm{df}$ & $\mathrm{S}^{2}$ & $F$ & $p$ \\
$\mathrm{~T}_{2}$ & 24.2256 & 19.8705 & 19.1425 & 18.6516 & 21.8906 & ${ }^{3} \mathrm{TE}$ & 3.6368 & 3 & 1.2123 & 40.3207 & 0.0001 \\
$\mathrm{~T}_{3}$ & 15.607 & 18.4464 & 17.0162 & 18.4303 & 17.2551 & ${ }^{4} \mathrm{pH}$ & 0.106 & 3 & 0.0353 & 1.1751 & 0.3346 \\
$\mathrm{~T}_{4}$ & 16.8242 & 18.5519 & 20.3251 & 19.7346 & 18.33 & ${ }^{5} \mathrm{MV}$ & 0.4944 & 3 & 0.1648 & 5.4815 & 0.0037 \\
$\bar{x}_{1}$ & 1.6082 & 1.5905 & 1.6226 & 1.5949 & 1.5399 & ${ }^{6} \mathrm{RS}$ & 0.0837 & 3 & 0.0279 & 0.9278 & 0.4386 \\
$\bar{x}_{2}$ & 2.0188 & 1.6559 & 1.5952 & 1.5543 & 1.8242 & ${ }^{7} \mathrm{FT}$ & 1.01 & 3 & 0.3367 & 11.1977 & 0.0001 \\
$\bar{x}_{3}$ & 1.3006 & 1.5372 & 1.418 & 1.5359 & 1.4379 & error & 0.9621 & 32 & 0.0301 & & \\
$\bar{x}_{4}$ & 1.402 & 1.546 & 1.6938 & 1.6446 & 1.5275 & $F_{0.05}$ & 2.90 & & & & \\
$\mathrm{R}$ & 0.7182 & 0.1187 & 0.2757 & 0.1087 & 0.3863 & $F_{0.01}$ & 4.46 & & & & \\
\hline
\end{tabular}

${ }^{1}$ Salidroside, ${ }^{2}$ Source of Variation, ${ }^{3}$ Temperature, ${ }^{4}$ Initial $\mathrm{pH},{ }^{5}$ Medium volume, ${ }^{6}$ Rotation speed,

${ }^{7}$ Fermentation. $p \leqslant 0.05$ and $p \leqslant 0.01$ were considered statistically significant difference and extremely significant difference, respectively.

Table 3. Test of significant effects of five factors on fermentation for TYR production from Rac56 using Data Processing System.

\begin{tabular}{|c|c|c|c|c|c|c|c|c|c|c|c|}
\hline \multicolumn{12}{|c|}{${ }^{1}$ TYR } \\
\hline $\mathrm{T}_{1}$ & 13.7101 & 15.9705 & 15.8391 & 15.244 & 13.4073 & ${ }^{2} \mathrm{~V}$ & SS & $\mathrm{df}$ & $s^{2}$ & F & $p$ \\
\hline $\mathrm{T}_{2}$ & 19.664 & 16.223 & 15.9399 & 14.6122 & 13.4654 & ${ }^{3} \mathrm{TE}$ & 2.1205 & 3 & 0.7068 & 21.3276 & 0.0001 \\
\hline $\mathrm{T}_{3}$ & 15.1039 & 14.2837 & 14.0013 & 16.6605 & 17.3406 & ${ }^{4} \mathrm{pH}$ & 0.1871 & 3 & 0.0624 & 1.8822 & 0.1525 \\
\hline $\mathrm{T}_{4}$ & 13.319 & 15.3198 & 16.0167 & 15.2803 & 17.5837 & ${ }^{5} \mathrm{MV}$ & 0.2343 & 3 & 0.0781 & 2.3563 & 0.0903 \\
\hline $\bar{x}_{1}$ & 1.1425 & 1.3309 & 1.3199 & 1.2703 & 1.1173 & ${ }^{6} \mathrm{RS}$ & 0.1865 & 3 & 0.0622 & 1.8762 & 0.1535 \\
\hline $\bar{x}_{2}$ & 1.6387 & 1.3519 & 1.3283 & 1.2177 & 1.1221 & ${ }^{7} \mathrm{FT}$ & 1.3532 & 3 & 0.4511 & 13.6102 & 0.0001 \\
\hline $\bar{x}_{3}$ & 1.2587 & 1.1903 & 1.1668 & 1.3884 & 1.4451 & error & 1.0605 & 32 & 0.0331 & & \\
\hline $\bar{x}_{4}$ & 1.1099 & 1.2767 & 1.3347 & 1.2734 & 1.4653 & $F_{0.05}$ & 2.90 & & & & \\
\hline $\mathrm{R}$ & 0.5288 & 0.1616 & 0.1679 & 0.1707 & 0.3480 & $F_{0.01}$ & 4.46 & & & & \\
\hline
\end{tabular}

Table 4. Results of an orthogonal-design experiment to determine the optimal fermentation conditions for SAL and TYR production from Rac56 using Data Processing System.

\begin{tabular}{cccccccc}
\hline \multirow{2}{*}{ Codes } & \multicolumn{5}{c}{ Factors } & & \multicolumn{2}{c}{ Content (mg/mL) } \\
\cline { 2 - 7 } & TE $^{\mathbf{1}}$ & $\mathbf{p H}^{\mathbf{2}}$ & $\mathbf{M V}^{\mathbf{3}}$ & $\mathbf{R S}^{\mathbf{4}}$ & FT $^{\mathbf{5}}$ & SAL $^{\mathbf{6}}$ & TYR $^{\mathbf{7}}$ \\
\hline 1 & 20 & 5 & 25 & 100 & 3 & $1.6264 \pm 0.1482$ & $1.0312 \pm 0.0071$ \\
2 & 20 & 6 & 50 & 150 & 7 & $1.9082 \pm 0.0720$ & $1.0128 \pm 0.0069$ \\
3 & 20 & 7 & 100 & 200 & 11 & $1.2076 \pm 0.1359$ & $1.1833 \pm 0.0524$ \\
4 & 20 & 8 & 150 & 250 & 15 & $1.6904 \pm 0.0822$ & $1.3428 \pm 0.2233$ \\
5 & 25 & 5 & 50 & 200 & 15 & $1.9383 \pm 0.0475$ & $2.0018 \pm 0.0009$ \\
6 & 25 & 6 & 25 & 250 & 11 & $2.0502 \pm 0.0477$ & $1.8792 \pm 0.0860$ \\
7 & 25 & 7 & 150 & 100 & 7 & $2.3392 \pm 0.1093$ & $1.4064 \pm 0.1196$ \\
8 & 25 & 8 & 100 & 150 & 3 & $1.7475 \pm 0.0789$ & $1.2673 \pm 0.0111$ \\
9 & 30 & 5 & 100 & 250 & 7 & $1.4483 \pm 0.1551$ & $1.0020 \pm 0.0014$ \\
10 & 30 & 6 & 150 & 200 & 3 & $1.3964 \pm 0.0129$ & $1.3012 \pm 0.1997$ \\
11 & 30 & 7 & 25 & 150 & 15 & $1.2126 \pm 0.1286$ & $1.3021 \pm 0.2278$ \\
12 & 30 & 8 & 50 & 100 & 11 & $1.1450 \pm 0.1473$ & $1.4293 \pm 0.2049$ \\
13 & 35 & 5 & 150 & 150 & 11 & $1.3489 \pm 0.2708$ & $1.2885 \pm 0.1504$ \\
14 & 35 & 6 & 100 & 100 & 15 & $1.2687 \pm 0.1773$ & $1.2145 \pm 0.1107$ \\
15 & 35 & 7 & 50 & 250 & 3 & $1.3894 \pm 0.0478$ & $0.8694 \pm 0.0961$ \\
16 & 35 & 8 & 25 & 200 & 7 & $1.6011 \pm 0.0927$ & $1.0672 \pm 0.0888$ \\
\hline
\end{tabular}

Values are mean $\pm \mathrm{SD} .{ }^{1}$ Temperature, ${ }^{2}$ Initial $\mathrm{pH},{ }^{3}$ Medium volume, ${ }^{4}$ Rotation speed, ${ }^{5}$ Fermentation time, ${ }^{6}$ salidroside, ${ }^{7}$ p-tyrosol. 


\section{Discussion}

Endophytic fungi are increasingly being recognized for their huge potential in the production of biologically active compounds for food, agriculture, and medicine [22]. In the present study, four endophytic fungi, namely, Rac12, Rct30, Rac56, and Rac63, were found to produce SAL or TYR. They were identified as Lachnum sp., Neonectriaramulariae and P. fortinii, respectively. Previously these endophytic fungi were also isolated from other plants and found to possess versatile capacities of producing biologically active compounds $[17,23,24]$. However, this study is the first to report the presence of these fungi in Rhodiola spp. and to reveal the existence of fungal endophytes with SAL- or TYR-producing abilities in nature. Taken together, our findings support the co-evolution theory of symbiosis between endophytic fungi and their hosts [9]. Furthermore, our findings provide a promising method of SAL and TYR production that is easy, sustainable, and low-cost, which are important factors not only in meeting the demands of SAL and TYR production using Rhodiola plants but also in protecting these medicinally important plant species.

Among the four fungi we studied, the Rac56 isolate exhibited the most stable and highest yield of SAL and TYR. By ITS sequence based genotyping we found our isolate Rac56 to be similar to P. fortinii, (Ascomycota, pezizomycotina, helotiales) a typical DSE fungus which is a widespread fungal endophyte of plants $[16,18]$. Several reports have shown that $P$. fortinii exhibits versatile capacities of promoting elemental nitrogen $(\mathrm{N})$ and phosphorus $(\mathrm{P})$ absorption by host plants [25] and synthesizing glucose [26] by co-existing with host roots. Further, P. fortinii have also been shown to aid the enhancement of disease resistance in hosts, controlling Verticillium yellows in Chinese cabbage [27], the capacity to produce chemicals such as hydroxamate siderophore [28] and other enzymes in improving host resistance and absorption of nutrients, for example, production of extracellular aryl sulfatase to decompose aryl sulphates [29]. All of these abilities, along with its ability to produce SAL and TYR, suggest that P. fortinii plays a key role in symbiosis with host plants.

SAL and TYR were originally derived from Rhodiola plants, and were synthesized from phenylalanine or tyrosine in the cinnamic acid pathway (originally called the shikimate pathway) [30]. TYR is synthesized first and serves as a precursor for SAL; in fact, SAL is the 8-O- $\beta$-D-glucoside of TYR and its glycosylation is dependent on UDP-glucosyltransferase catalysis [5,31]. As closely related secondary metabolites of the cinnamic acid pathway, SAL and TYR are always detected together, and their yields were found to be stable in Rhodiola plants [32]. However, it is not yet known whether the same pathway is involved in SAL and TYR biosynthesis in fungi and their hosts, or whether the genes involved in SAL and TYR biosynthesis were horizontally transferred from the host and probably mutated during the course of evolution, which may explain the stabilized or unstabilized yields of SAL and TYR after repeated sub-culturing in certain endophytic strains. Such questions are of significant interest for further elucidating the interaction between endophytic fungi and their hosts, and regulating SAL and TYR production efficiency and metabolic optimization.

\section{Materials and Methods}

\subsection{Plant Materials and Isolation of Endophytic Fungi}

R. crenulata samples were collected from Mila Pass (altitude $5007 \mathrm{~m}, \mathrm{E} 92^{\circ} 22^{\prime} 31^{\prime \prime}-92^{\circ} 24^{\prime} 88^{\prime \prime}$, N $\left.29^{\circ} 44^{\prime} 33^{\prime \prime}-29^{\circ} 46^{\prime} 56^{\prime \prime}\right)$, and R. sachalinensis and R. angusta were collected from Changbai Mountain (altitude $2200 \mathrm{~m}, \mathrm{E} 128^{\circ} 08^{\prime} 17^{\prime \prime}-128^{\circ} 11^{\prime} 10^{\prime \prime}, \mathrm{N} 44^{\circ} 11^{\prime} 20^{\prime \prime}-44^{\circ} 13^{\prime} 29^{\prime \prime}$ ) of China, and they were identified by Jinlong Cui and voucher specimens were deposited in the Institute of Applied Chemistry, Shanxi University. Isolation and purification of fungus from plant samples was done as described earlier in Sun et al. [12].

\subsection{Fermentation and Secondary Metabolite Extraction Fromendophytic Fungal spp.}

Six pieces ( $5 \mathrm{~mm}$ diameter) of mycelial agar plugs from the edge of growing cultures of endophytic fungi were each inoculated into $500 \mathrm{~mL}$ Erlenmeyer flask with $200 \mathrm{~mL}$ of liquid $\mathrm{CD}$ medium $\left(\mathrm{NaNO}_{3}\right.$, 
$3 \mathrm{~g} ; \mathrm{K}_{2} \mathrm{HPO}_{3}, 1 \mathrm{~g} ; \mathrm{MgSO}_{4} \cdot 7 \mathrm{H}_{2} \mathrm{O}, 0.5 \mathrm{~g} ; \mathrm{KCl}, 0.5 \mathrm{~g} ; \mathrm{FeSO}_{4}, 0.01 \mathrm{~g}$; sucrose, $30 \mathrm{~g}$; and deionized water to a total volume of $1000 \mathrm{~mL})$. All flasks were incubated under constant shaking $(150 \times g)$ at $25 \pm 2{ }^{\circ} \mathrm{C}$ for 10 days. The fungal broth was filtered to separate the culture broth and mycelia using Whatman No. 1 filter paper. The mycelia and filtrate were extracted following the SAL and TYR extraction method as described in Chinese Pharmacopoeia [33]. The mycelia were dried, powdered, weighed, and extracted three times with 10-fold $(w / v)$ methanol by ultrasonication for $30 \mathrm{~min}$, after which the three methanol extracts were combined. The culture filtrate was then evaporated under reduced pressure $\left(8 \times 10^{3} \mathrm{~Pa}\right)$ to yield an extract, which was weighed and extracted three times with 10-fold $(w / v)$ methanol for $10 \mathrm{~min}$ and extracts pooled. The extraction solution was then filtered using Whatman No. 1 filter paper and centrifuged at $5000 \times g$ for $10 \mathrm{~min}$. Lastly, the supernatants were harvested to obtain the mycelia and filtrate extracts.

\subsection{Screening of SAL and TYR-Producing Endophytic Fungi}

The mycelia and filtrate extracts prepared were filtered through a $0.45 \mu \mathrm{m}$ Millex-HV filter membrane (Millipore, Billerica, MA, USA) before determination of SAL and TYR contents by RP-HPLC analyses based on the method described in Chinese Pharmacopoeia [33]. The analytical HPLC system (Agilent 1200 LC Series, Santa Clara, CA, USA) was equipped with a quaternary pump with vacuum degasser, an Eclipse XDB- $\mathrm{C}_{18}(4.6 \times 250 \mathrm{~mm}, 5 \mu \mathrm{m}$, Agilent), a $10 \mu \mathrm{L}$ manual injector, a thermostatted column compartment, and a UV detector. The mobile phase consisted of a methanol/water mixture (30:70) at a flow rate of $1.0 \mathrm{~mL} / \mathrm{min}$ at $30^{\circ} \mathrm{C}$ and monitored using a UV detector at $276 \mathrm{~nm}$. HPLC-grade methanol was purchased from Fisher Scientific (Pittsburgh, PA, USA). Ultrapure water was prepared using an ultrapure water system from Arium Comfort I (Sartorius, Göttingen, Germany). The SAL and TYR standards were purchased from the National Institute for the Control of Pharmaceutical and Biological Products (Beijing, China). Detections of SAL and TYR in fungal extracts were based on their retention times. Quantifications of SAL and TYR were done using a linear regression equation fitted in a calibration graph as $\mathrm{y}=0.88325 \mathrm{x}+0.44806(\mathrm{r}=0.99951)$ and $\mathrm{y}=2.59700 \mathrm{x}+2.18000(\mathrm{r}=0.99960)$, respectively. The concentration ranges of SAL and TYR were $2 \mu \mathrm{g} / \mathrm{mL}$ to $200 \mu \mathrm{g} / \mathrm{mL}$ and $1.6 \mu \mathrm{g} / \mathrm{mL}$ to $160 \mu \mathrm{g} / \mathrm{mL}$, respectively.

\subsection{Molecular Weight Determination by UPLC/Q-TOF-MS}

The identity of SAL and TYR produced by the fungi was confirmed by determination of their molecular masses using ultra-performance liquid chromatography/quadrupole time-of-flight mass spectrometry (UPLC/Q-TOF-MS) analyses. Analytical UPLC separation was performed on a Waters Acquity I-Class system (Waters Corp., Milford, MA, USA), equipped with a UV detector, a BEH $C_{18}$ column $(2.1 \times 100 \mathrm{~mm}, 1.7 \mu \mathrm{m})$, a quaternary pump, and an autosampler. The mobile phase contained two solutions, namely (A) $0.1 \%(v / v)$ formic acid in high-purity water and (B) chromatographic grade acetonitrile. A timed gradient program of $\mathrm{T}(\mathrm{min}) / \mathrm{B}(\%)(0 / 5,9 / 35,12 / 70,12.5 / 5,15 / 5)$ was used for elution, with a flow rate of $0.4 \mathrm{~mL} / \mathrm{min}$. The column temperature was maintained at $40{ }^{\circ} \mathrm{C}$, the sample injection volume was $3 \mu \mathrm{L}$, and the detection wavelength was set to $277 \mathrm{~nm}$. MS analyses were performed on a Waters Xevo G2 Q-TOF (Micromass MS Technologies, Manchester, UK), equipped with an electrospray ionization source. Lock Spray ${ }^{\mathrm{TM}}$ was used to ensure mass accuracy and reproducibility. The deprotonated leucine-enkephalin $\left(\mathrm{C}_{28} \mathrm{H}_{37} \mathrm{~N}_{5} \mathrm{O}_{7}\right)$ at $\mathrm{m} / z 554.2615$ was used as the lock mass in negative-ion detection mode. The source block and desolvation lamp were kept at $100{ }^{\circ} \mathrm{C}$ and $400{ }^{\circ} \mathrm{C}$, respectively. The nebulization gas was set to $800 \mathrm{~L} / \mathrm{h}$ at $400^{\circ} \mathrm{C}$. The cone gas was $20 \mathrm{~L} / \mathrm{h}$, and the source temperature was $100^{\circ} \mathrm{C}$. The capillary voltage and cone voltage were set at $2700 \mathrm{~V}$ and $40 \mathrm{~V}$, respectively. The collision energy was $6 \mathrm{~V}$, and the MS data were acquired from a mass-to-charge ratio $(\mathrm{m} / \mathrm{z})$ range of 100-1500. MS/MS experiments were performed by ramping collision energies from $35 \mathrm{~V}$ to $45 \mathrm{~V}$. A dwelling time of $0.2 \mathrm{~s}$ was employed with an inter-acquisition delay of $0.01 \mathrm{~s}$. Data acquisition and processing were performed using Masslynx v 4.1 software (Version 9.50; Science Press, Beijing, China). 


\subsection{Structure Determination of SAL and TYR in Fungal Extracts by ${ }^{1} H-N M R$}

To confirm the authenticity of fungal SAL and TYR, ${ }^{1} \mathrm{H}$ nuclear magnetic resonance $\left({ }^{1} \mathrm{H}-\mathrm{NMR}\right)$ spectra were determined on dried fungal extracts dissolved in methanol- $d_{4}\left(\mathrm{CD}_{3} \mathrm{OD}\right)$ using an AVANCE $300 \mathrm{MHz}$ spectrometer (Bruker, Fallanden, Switzerland). Chemical shifts were reported as $\delta$ values relative to tetramethylsilane used as an internal reference. Spectra related compounds were assigned by comparing fungal extracts and authentic SAL and TYR in $\mathrm{CD}_{3} \mathrm{OD}$ following the method in reference $[4,21]$.

\subsection{Identification of SAL and TYR-Producing Fungus}

Morphological identification of SAL and TYR-producing fungi was based on colony appearance and sporulation. Mycelial characteristics, such as color, septum, transparency, shape, and sporogenous structure and spores' characteristics, such as shape, size, and conidial fructification, were observed through specimens mounting in $2 \% \mathrm{KOH}$ under a light microscope (BX53, Olympus, Tokyo, Japan). To authenticate the results of morphological identification, molecular method was adopted to verify the taxonomic status of fungi. The ITS sequence (ITS1: TCCGTAGGTGAACCTGCGG; ITS4: TCCTCCGCTTATTGATATGC) was subjected to sequence similarity search performed on the NCBI database (http:/ / www.ncbi.nlm.gov/BLAST). Sequences with high homology with the submitted isolate were retrieved and analyzed using Clustal $\mathrm{W}$, and a neighbor-joining (NJ) phylogenetic tree was constructed from the evolutionary distance data using MEGA 4.0. The ITS rDNA sequences of the Rac12, Rac30, Rac56, and Rac63 strains that were identified by the molecular method are deposited in GenBank under accession numbers KJ542268, KJ542219, KJ542292, and KJ542358, respectively.

\subsection{Optimization of Fermentation Conditions for $S A L$ and TYR Production from Rac56}

Single-factor design was employed to evaluate the effects of four culture media: CD, PD (potato, $200 \mathrm{~g}$; glucose, $20 \mathrm{~g}$; deionized water, $1000 \mathrm{~mL}$ ), SD (peptone, $10 \mathrm{~g}$; glucose, $40 \mathrm{~g}$; deionized water, $1000 \mathrm{~mL}$ ), and LBP(lactose, $5 \mathrm{~g}$; beef extract, $5 \mathrm{~g}$; yeast extract, $5 \mathrm{~g}$; peptone, $10 \mathrm{~g}$; glucose, $10 \mathrm{~g}$; $\mathrm{NaCl}$, $5 \mathrm{~g}$; deionized water, $1000 \mathrm{~mL} ; \mathrm{pH} 6.8 \pm 0.2$ ) on SAL and TYR production by Rac56. An orthogonal design of five factors and four levels (L16 $\left(4^{5}\right)$ ) was adopted to optimize the culture conditions for SAL and TYR production using Data Processing System software (Version 9.50; Science Press, Beijing, China), as shown in Table 5. The fermentation broths were extracted, and SAL and TYR yields were determined to obtain the optimal fermentation parameters for an increased SAL and TYR yield based on the methods outlined in this study.

Table 5. Five factors and their four levels in an L16 $\left(4^{5}\right)$ orthogonal design.

\begin{tabular}{ccccc}
\hline \multirow{2}{*}{ Factor } & \multicolumn{4}{c}{ Levels } \\
\cline { 2 - 5 } & $\mathbf{1}$ & $\mathbf{2}$ & $\mathbf{3}$ & $\mathbf{4}$ \\
\hline Temperature $\left({ }^{\circ} \mathrm{C}\right)$ & 20 & 25 & 30 & 35 \\
Initial $\mathrm{pH}$ & 5 & 6 & 7 & 8 \\
Medium volume $(\mathrm{mL})$ & 25 & 50 & 100 & 150 \\
Rotation speed $(\mathrm{rpm})$ & 100 & 150 & 200 & 250 \\
Fermentation time (day) & 3 & 7 & 11 & 15 \\
\hline
\end{tabular}

\subsection{Statistical Analysis}

All experiments were performed in triplicate and data are presented as mean \pm standard deviation (SD). Statistical difference was analyzed with multi-way ANOVA using DPS 9.50 software (Science Press, Beijing, China).

Supplementary Materials: Supplementary materials can be accessed at: http://www.mdpi.com/1420-3049/21/ 4/502/s1. 
Acknowledgments: We would like to thank the financial support from National Natural Sciences Foundation of China (No. 31270383), Natural Science Foundation of Shanxi Province, China (No. 2014011029-1), Specialized Research Fund for the Doctoral Program of Higher Education of China (No. 20121401120001), and Forestry Public Industry Scientific Research (201304326). We also thank Vinod Vijayakumar (The Ohio State University) for reading and correcting the manuscript.

Author Contributions: Jinlong Cui and Mengliang Wang conceived and designed the experiment. Jinlong Cui, Tingting Guo and Jianbin Chao performed the experiments. Junhong Wang contributed reagents and materials. Jinlong Cui wrote the paper.

Conflicts of Interest: The authors declare no conflict of interest.

\section{Abbreviations}

The following abbreviations are used in this manuscript:

$\begin{array}{ll}\text { SAL } & \text { salidroside } \\ \text { YAL } & p \text {-tyrosol } \\ \text { DSE } & \text { dark septate endophytes } \\ \text { CD } & \text { Czapek-Dox medium } \\ \text { PD } & \text { potato dextrose medium } \\ \text { SD } & \text { sabouraud medium } \\ \text { LBP } & \text { lactose-beef extract-peptone medium } \\ \text { HPLC } & \text { high performance liquid chromography } \\ \text { UPLC/Q-TOF-MS } & \text { ultra-performance liquid chromatography/quadrupole time-off light mass spectrometry } \\ { }^{1} \mathrm{H}-\mathrm{NMR} & { }^{1} \mathrm{H} \text { nuclear magnetic resonance }\end{array}$

\section{References}

1. Panossian, A.; Hamm, R.; Wikman, G.; Efferth, T. Mechanism of action of Rhodiola, salidroside, tyrosol and triandrin in isolated neuroglial cells: An interactive pathway analysis of the downstream effects using RNA microarray data. Phytomedicine 2014, 21, 1325-1348. [CrossRef] [PubMed]

2. Hung, S.K.; Perry, R.; Ernst, E. The effectiveness and efficacy of Rhodiola rosea L.: A systematic review of randomized clinical trials. Phytomedicine 2011, 18, 235-244. [CrossRef] [PubMed]

3. Chiang, H.M.; Chen, H.C.; Wu, C.S.; Wu, P.Y.; Wen, K.C. Rhodiola plants: Chemistry and biological activity. J. Food Drug Anal. 2015, 23, 359-369. [CrossRef]

4. Peschel, W.; Prieto, J.M.; Karkour, C.; Williamson, E.M. Effect of provenance, plant part and processing on extract profiles from cultivated European Rhodiola rosea L. for medicinal use. Phytochemistry 2013, 86, 92-102. [CrossRef] [PubMed]

5. Yu, H.S.; Ma, L.Q.; Zhang, J.X.; Shi, G.L.; Hu, Y.H.; Wang, Y.N. Characterization of glycosyltransferases responsible for salidroside biosynthesis in Rhodiola sachalinensis. Phytochemistry 2011, 72, 862-870. [CrossRef] [PubMed]

6. Petrini, O.; Sieber, T.N.; Toti, L.; Viret, O. Ecology, metabolite production, and substrate utilization in endophytic fungi. Nat. Toxins 1992, 1, 185-196. [CrossRef] [PubMed]

7. Strobel, G.; Daisy, B. Bioprospecting for microbial endophytes and their natural products. Microbiol. Mol. Biol. R. 2003, 67, 491. [CrossRef]

8. Rodriguez, R.J.; White, J.F.; Arnold, A.E.; Redman, R.S. Fungal endophytes: Diversity and functional roles. New Phytol. 2009, 182, 314-330. [CrossRef] [PubMed]

9. Tan, R.X.; Zou, W.X. Endophytes: A rich source of functional metabolites. Nat. Prod. Rep. 2001, 18, 448-459. [CrossRef] [PubMed]

10. Xu, X.H.; Su, Z.Z.; Wang, C.; Kubicek, C.P.; Feng, X.X.; Mao, L.J.; Wang, J.Y.; Chen, C.; Lin, F.C.; Zhang, C.L. The rice endophyte Harpophora oryzae genome reveals evolution from a pathogen to a mutualistic endophyte. Sci. Rep. UK 2014, 4. [CrossRef] [PubMed]

11. Rodriguez, R.J.; Henson, J.; Van Volkenburgh, E.; Hoy, M.; Wright, L.; Beckwith, F.; Kim, Y.O.; Redman, R.S. Stress tolerance in plants via habitat-adapted symbiosis. Isme J. 2008, 2, 404-416. [CrossRef] [PubMed] 
12. Sun, S.S.; Zeng, X.; Zhang, D.W.; Guo, S.X. Diverse fungi associated with partial irregular heartwood of Dalbergia odorifera. Sci. Rep. UK 2015, 5. [CrossRef] [PubMed]

13. Stierle, A.; Strobel, G.; Stierle, D. Taxol and taxane production by taxomyces-andreanae, an endophytic fungus of pacific yew. Science 1993, 260, 214-216. [CrossRef] [PubMed]

14. Nicoletti, R.; Ferranti, P.; Caira, S.; Misso, G.; Castellano, M.; di Lorenzo, G.; Caraglia, M. Myrtucommulone production by a strain of Neofusicoccum australe endophytic in myrtle (Myrtus communis). World J. Microbiol. Biotechnol. 2014, 30, 1047-1052. [CrossRef] [PubMed]

15. El-Hawary, S.S.; Mohammed, R.; AbouZid, S.F.; Bakeer, W.; Ebel, R.; Sayed, A.M.; Rateb, M.E. Solamargine production by a fungal endophyte of Solanum nigrum. J. Appl. Microbiol. 2016, 120, 900-911. [CrossRef] [PubMed]

16. Addy, H.D.; Hambleton, S.; Currah, R.S. Distribution and molecular characterization of the root endophyte Phialocephala fortinii along an environmental gradient in the boreal forest of Alberta. Mycol. Res. 2000, 104, 1213-1221. [CrossRef]

17. Brenn, N.; Menkis, A.; Grunig, C.R.; Sieber, T.N.; Holdenrieder, O. Community structure of Phialocephala fortinii s. lat. in European tree nurseries, and assessment of the potential of the seedlings as dissemination vehicles. Mycol. Res. 2008, 112, 650-662. [CrossRef] [PubMed]

18. Grunig, C.R.; Brunner, P.C.; Duo, A.; Sieber, T.N. Suitability of methods for species recognition in the Phialocephala fortinii-Acephala applanata species complex using DNA analysis. Fungal Genet. Biol. 2007, 44, 773-788. [CrossRef] [PubMed]

19. Wang, C.J.K.; Wilcox, H.E. New species of ectendomycorrhizal and pseudomycorrhizal fungiPhialophora-Finlandia, Chloridium-Paucisporum, and Phialocephala-Fortinii. Mycologia 1985, 77, 951-958. [CrossRef]

20. Wilson, B.J.; Addy, H.D.; Tsuneda, A.; Hambleton, S.; Currah, R.S. Phialocephala sphaeroides sp nov. a new species among the dark septate endophytes from a boreal wetland in Canada. Can. J. Bot. 2004, 82, 607-617. [CrossRef]

21. Ioset, K.N.; Nyberg, N.T.; Van Diermen, D.; Malnoe, P.; Hostettmann, K.; Shikov, A.N.; Jaroszewski, J.W. Metabolic profiling of Rhodiola rosea rhizomes by H-1 NMR spectroscopy. Phytochem. Anal. 2011, 22, 158-165. [CrossRef] [PubMed]

22. Aly, A.H.; Debbab, A.; Proksch, P. Fungal endophytes: Unique plant inhabitants with great promises. Appl. Microbiol. Biotechnol. 2011, 90, 1829-1845. [CrossRef] [PubMed]

23. Shiono, Y.; Shimanuki, K.; Hiramatsu, F.; Koseki, T.; Tetsuya, M.; Fujisawa, N.; Kimura, K. Pyrrospirones A and B, apoptosis inducers in HL-60 cells, from an endophytic fungus, Neonectria ramulariae Wollenw KS-246. Bioorg. Med. Chem. Lett. 2008, 18, 6050-6053. [CrossRef] [PubMed]

24. Zhuang, W.Y. A new species of Lachnum on leaves of Livistona and a key to the Chinese species of the genus. Mycotaxon 2003, 86, 375-382.

25. Jumpponen, A.; Trappe, J.M. Dark septate endophytes: A review of facultative biotrophic root-colonizing fungi. New Phytol. 1998, 140, 295-310. [CrossRef]

26. Jumpponen, A.; Trappe, J.M. Performance of Pinus contorta inoculated with two strains of root endophytic fungus, Phialocephala fortinii: Effects of synthesis system and glucose concentration. Can. J. Bot. 1998, 76, 1205-1213. [CrossRef]

27. Narisawa, K.; Usuki, F.; Hashiba, T. Control of verticillium yellows in Chinese cabbage by the dark septate endophytic fungus LtVB3. Phytopathology 2004, 94, 412-418. [CrossRef] [PubMed]

28. Bartholdy, B.A.; Berreck, M.; Haselwandter, K. Hydroxamate siderophore synthesis by Phialocephala fortinii, a typical dark septate fungal root endophyte. Biometals 2001, 14, 33-42. [CrossRef] [PubMed]

29. Addy, H.D.; Piercey, M.M.; Currah, R.S. Microfungal endophytes in roots. Can. J. Bot. 2005, 83, 1-13. [CrossRef]

30. Zhang, J.X.; Ma, L.Q.; Yu, H.S.; Zhang, H.; Wang, H.T.; Qin, Y.F.; Shi, G.L.; Wang, Y.N. A tyrosine decarboxylase catalyzes the initial reaction of the salidroside biosynthesis pathway in Rhodiola sachalinensis. Plant Cell Rep. 2011, 30, 1443-1453. [CrossRef] [PubMed]

31. Ma, L.Q.; Liu, B.Y.; Gao, D.Y.; Pang, X.B.; Lu, S.Y.; Yu, H.S.; Wang, H.; Yan, F.; Li, Z.Q.; Li, Y.F.; Ye, H.C. Molecular cloning and overexpression of a novel UDP-glucosyltransferase elevating salidroside levels in Rhodiola sachalinensis. Plant Cell Rep. 2007, 26, 989-999. [CrossRef] [PubMed] 
32. Gyorgy, Z.; Tolonen, A.; Neubauer, P.; Hohtola, A. Enhanced biotransformation capacity of Rhodiola rosea callus cultures for glycosid production. Plant Cell Tiss Org. 2005, 83, 129-135. [CrossRef]

33. Commission, C.P. Pharmacopoeia of the People's Republic of China; Chinese Medical Science and Technology Press: Beijing, China, 2010; p. 144.

Sample Availability: Samples of the compoundsare not available from the authors.

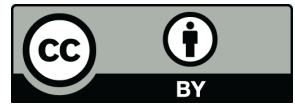

(C) 2016 by the authors; licensee MDPI, Basel, Switzerland. This article is an open access article distributed under the terms and conditions of the Creative Commons Attribution (CC-BY) license (http:/ / creativecommons.org/licenses/by/4.0/). 